\title{
Purchase Contract Management with Demand Forecast Updates
}

\author{
Hongyan Huang \\ Dept. of Systems Engineering and Engineering Management \\ The Chinese University of Hong Kong, Shatin, Hong Kong \\ and \\ Suresh P. Sethi Houmin Yan \\ School of Management \\ The University of Texas at Dallas, Richardson, TX 75083-0688
}

September 20, 2004

\begin{abstract}
We study a two-stage purchase contract with a demand forecast update. The purchase contract provides the buyer an opportunity to adjust an initial commitment based on an updated demand forecast obtained at a later stage. An adjustment, if any, incurs a fixed as well as a variable cost. Using a dynamic programming formulation, we obtain optimal solutions for a class of demand distributions. We also discuss how these results can be applied to gain managerial insights that help in making decisions regarding where to allocate efforts in improving the forecast quality and whether or not to sign a contract.
\end{abstract}

\section{Introduction}

In this paper we study a buyer's problem involving a purchase contract with a demand forecast update. Because of the presence of a lead time, the buyer makes an initial purchase decision with a preliminary demand forecast. The buyer is aware that an improved demand forecast will be available at a later stage. The purchase contract provides the buyer an opportunity to modify his initial order by exercising an option: the buyer may exercise the option at a fixed cost, and then increase the initial order quantity (resp. cancel some ordered items) at a higher unit cost (resp. for a lower-than-cost refund). Our objective is to obtain an optimal policy which describes the initial order quantity and a reaction plan to the improved demand forecast available at the later stage. 
This research is motivated by micro-controller chip purchase activities in a Hong Kong security equipment company. The company produces various security devices for industrial, residential, and military applications worldwide. The micro-controllers are the key components for its products. When micro-controllers are ordered from a semiconductor manufacturer, the company is allowed to specify the data (programming code) in the micro-controllers with read-only memory $(\mathrm{ROM})$. The process is known as customer masking in wafer fabrication, which requires a sufficient lead time (typically 16-20 weeks). The fundamental difficulty the security equipment company faces lies in not being able to know the demand until a time close to delivery. In addition, the company cannot afford to keep high volume inventory in micro-controllers for a number of reasons, such as short product life cycles, frequent engineering changes, and a sharply decreasing price trend in semiconductor products. One way of dealing with the difficulty is to use a general purpose, albeit more expensive, micro-controller with erasable programmable read-only memory (EPROM). Another option is a form of agreement that can be reached between the supplier and the buyer, which allows the buyer to change its initial order quantity with both fixed and variable costs before a specific time. This is known as a purchase contract with volume flexibility. For high uncertainty products, the company has adopted our approach in evaluating and executing purchase contracts for its customer masking chips with one of its major micro-controller manufacturers. At the same time, for stable products, the company uses a combination of one-time programmable and EPROM chips when the demand cannot be satisfied by the customer masking chips.

We formulate the problem as a two-stage dynamic programming problem, where the decisions are the initial order quantity and the reaction plan which specifies how to adjust the initial order in view of the improved demand information obtained at Stage 2. The optimal policy at Stage 2 for increasing or decreasing the initial order can be expressed in terms of their respective $(s, S)$ parameters. When the demand signal is the location parameter of the conditional demand distribution and the conditional demand given the signal is a $\mathrm{PF}_{2}$ density, we prove that the total expected cost is unimodal with respect to the initial order quantity. Therefore, the initial optimal policy is a base-stock policy with a closed-form solution for the base-stock level. In the case of uniform conditional distribution of demand, we discuss how the results obtained enable 
us to perform a sensitivity analysis with respect to changes in forecast updating parameters.

Our model could be considered as a two-stage extension of the classical newsvendor problem to allow for a contract, a fixed cost, a forecast update, and a possibility of the initial order adjustment, while, at the same time, preserving the explicitness of the solution - a very appealing feature of the newsvendor problem. The explicit form of the solution allows us to obtain a number of valuable insights into better purchase contract management. When there are other means of hedging demand uncertainties such as product substitution, we establish the value of the purchase contract. We prove that the optimal cost function is monotone with respect to the contract exercise cost. In addition, we demonstrate the asymptotic property of the cost function, namely, that the cost converges to a fixed value when the contract exercise cost is sufficiently large. These findings provide benchmarks in determining strategies for hedging demand uncertainties.

The paper is organized as follows. Section 2 provides a brief review of the literature, and discusses how our model compares or contrasts with the literature. Section 3 introduces the notation and formulates the problem. After a discussion of factors that are involved in decision making, we formulate the problem of the optimal initial order policy at Stage 1 and the optimal adjustment policy at Stage 2 as a two-stage dynamic programming problem. In Section 4, we prove that the optimal policy at Stage 2 is a generalized $(s, S)$ policy for general demand distributions. While the general problem at Stage 1 can be solved numerically, we provide solutions for the class of $\mathrm{PF}_{2}$ distributions in Section 5. In Section 6, we provide a sensitivity analysis, which aids in deciding on the investment the buyer can make in improving the forecast update and contract parameters. Moreover, it is also possible to determine a critical contract exercise price above which the contract is not as desirable as an available hedging alternative. Section 7 concludes the paper. The appendix contains proofs of results.

\section{Review of and Relationship with the Literature}

There are a number of papers in the literature dealing with problems of uncertain demands with forecast updates. Murray and Silver (1966) use a Bayesian approach to estimate the demand. Lovejoy (1990) develops a myopic policy with a parameterized adaptive demand process. Sethi and Sorger (1991) develop a dynamic programming framework for rolling horizon decision making 
with forecast updates obtained at some cost. They formulate a dynamic stochastic production planning problem which involves determination of the optimal length of the forecast window and the optimal production quantity in each period. Their objective is minimization of the total cost of production, inventory and forecasting over a given planning horizon.

Fisher and Raman (1996) discuss the problem of an accurate response to uncertain demand when some information about early sales is available. The problem of optimal production commitments is formulated as a stochastic programming problem. Constraints on the production capacity and the minimum lot size are considered. The results are applied to a major sportswear company, and it is reported to have resulted in remarkable savings. In a special case with bivariate normally distributed demand forecasts and equal under- and over-production costs, the authors are able to provide a closed-form solution to the problem under consideration.

Motivated by quick response programs in the apparel industry, Iyer and Bergen (1997) divide the planning horizon into two stages. Information obtained in the first stage is used to update the parameters in the demand distribution function by an application of the Bayesian approach. The decision of how much to produce is made only in the second stage. The model allows one to evaluate the relationship between buyers and manufacturers. Effects of quick response on the service levels, price, and volume are also discussed.

Yan, Liu and Hsu (2003) develop a dual supply model with one demand forecast update as a two-stage dynamic programming problem. The decision variables are the order quantities in the first and the second stage. Under the assumption of uniformly distributed demand forecasts and increasing purchase costs over time, they demonstrate that the total cost is convex with respect to the initial order quantity. They also prove that the optimal policy is myopic if the demand processes satisfy some regularity conditions.

Sethi, Yan and Zhang (2001) consider a periodic review inventory model - termed an onion layer peeling model - with fast and slow delivery modes and multiple demand forecast updates, where fast and slow orders are delivered at the end of the current and the next periods, respectively. Both finite and infinite horizon problems are treated. They show that a modified base-stock policy is optimal, where the inventory position relevant for the slow order includes the amount ordered via the fast mode. Sethi, Yan and Zhang (2003) extend the model by allowing 
for a fixed ordering cost, and show that an $(s, S)$-type policy is optimal for both fast and slow orders.

Traditional inventory models assume a simple buyer-supplier arrangement. The buyer places an order at any time for any amount at a fixed cost and a given unit price, and the supplier provides the product. However, this results in a great deal of uncertainty for both parties, since very little is known about the eventual demand at the time of the order. In many industries, forms of arrangements known as contracts exist to strike a balance between flexibility and uncertainty. Bassok and Anupindi (2002) model a contract with both total minimum dollar volume commitment and flexibility to adjust the volume of individual products. In addition, Anupindi and Bassok (1998) analyze a supply contract with various types of flexibilities. Eppen and Iyer (1997) discuss a so-called backup agreement in the fashion industry for a catalog company. It entails that the supplier holds back a constant fraction of the commitment and delivers the remaining units to the catalog company before the start of the fashion season. It allows the catalog company to make decisions after observing the early demand. That is, the company may order up to the backup quantity at the original cost, along with a penalty cost for any backup units that are not ordered. They find that a backup agreement has an impact on the expected profit.

Barnes-Schuster, Bassok and Anupindi (2002) study the role of a supply contract between the buyer and the supplier in a two-stage model. In their model, the option of volume flexibility applies to the second stage. Structural properties of the objective functions for both the buyer and the supplier are explored. They show that in order to achieve channel coordination, the contract exercise price must be in the form of a piecewise linear function. The contract option price is also evaluated numerically. Donohue (2000) considers a supply contract as a risk-sharing mechanism between the buyer and the supplier. She focuses on channel coordination by determining the wholesale price and the return policy. Gurnani and Tang (1999) investigate optimal ordering policies for a two-stage model, where the demand information is updated between Stages 1 and 2. They provide a nested newsvendor model for determining the optimal order quantity for each stage. In the case of a bivariate normally distributed demand, Gurnani and Tang provide an explicit solution for the special cases of the worthless and the perfect information update. Cachon (2003) reviews and extends the literature of supply chain coordination, addressing the 
coordination issues of the two-stage newsvendor. The newsvendor is allowed only to increase, at the second stage, the original order at a higher unit cost and no fixed cost. It is found that it is possible to coordinate the supply chain with a buy-back contract.

We conclude this section by a discussion of how our model is related to the literature. In contrast with Eppen and Iyer (1997), we allow the buyer to adjust the initial order at the second stage. Whereas Sethi, Yan and Zhang (2001) and Yan, Liu and Hsu (2003) focus on the optimal order quantities from two supply modes - fast (expensive) and slow (cheap) — with demand forecast updates, we look into how a purchase contract affects the buyer's decision. In contrast to Sethi, Yan and Zhang (2003), we allow in Stage 2 for cancellation of a part of the initial order issued in Stage 1. It is important to observe that the $K$-convexity of the cost function is not preserved under the order cancellation feature. In view of this, we specialize our distribution to be $\mathrm{PF}_{2}$ so as to ensure a unimodal cost function. Our work differs from Barnes-Schuster, Bassok and Anupindi (2002) two ways. We update both the mean and the spread of the demand forecast whereas Barnes-Schuster, Bassok and Anupindi update only the minimum demand. In addition, we consider a fixed contract exercise cost and use the contract to hedge the demand uncertainty. In comparison with the models of Donohue (2000) and Gurnani and Tang (1999), we consider a fixed contract exercise cost and obtain an explicit solution for any degree of the demand information update. The worthless and perfect information updates are, therefore, special cases of our model.

\section{Problem Formulation}

A purchase contract is an agreement between a seller and a buyer. The contract specifies terms of purchase in two stages and delivery. In the first stage, the buyer places an initial order. In the second stage, based on the improved demand forecast and the decision made in the first stage, the buyer may adjust the initial order upward at a cost no less than the initial cost, or downward with a refund value that is lower than the initial cost. In addition, a fixed exercise cost is also incurred if any adjustment is made. The items with the confirmed quantity at Stage 2 are delivered at the end of the second stage.

Specifically, the buyer faces the following cost parameters: a cost of $c_{1}>0$ per unit for items 
ordered at Stage 1 and a cost of $c_{21}>0$ per unit for items ordered at Stage 2. On the other hand, if a unit of the Stage 1 order is cancelled at Stage 2, it is modeled as a negative order at Stage 2. In this case, the buyer has either a refund value or must pay a cancellation cost. We model this situation by letting $c_{22}$ denote the refund value or the cancellation cost per unit at Stage 2, where $c_{22}$ is the unit refund when $c_{22} \geq 0$ and $-c_{22}$ is the unit cancellation cost when $c_{22}<0$. This phenomenon of cancellation cost is common when the merchandise is perishable or hazardous. It is reasonable to assume $c_{21} \geq c_{1} \geq c_{22}$. In addition, there is a fixed contract exercise cost $K \geq 0$ at Stage 2 for adjustment to the initial order quantity. Furthermore, we assume a unit shortage cost of $p>c_{21}$ for unsatisfied demand since otherwise it would be optimal for the buyer not to order additional items at Stage 2. A positive unit holding (or salvage) cost of $h>-c_{22}$ is charged for excess inventory. Note that $h>-c_{22}$ is always true when $c_{22} \geq 0$. However, when $c_{22}<0$, the unit holding cost must be more expensive than the unit cancellation cost $-c_{22}$, otherwise it would be optimal not to cancel any part of the initial order. We leave out two trivial cases, $p=c_{21}$ and $h=-c_{22}>0$, for which the optimal solution is straightforward, it ignores any adjustment.

Let $D \geq 0$ represent the random demand in Stage 2 with density $f_{D}(\cdot)$. Let $\Psi \geq 0$ represent a signal observed in Stage 1 with distribution $F_{\Psi}(\cdot)$ and density $f_{\Psi}(\cdot)$. Let $F_{D \mid \psi}(\cdot)$ denote the cumulative distribution function of $D$ given $\Psi=\psi$ with $f_{D \mid \psi}(\cdot)$ as the corresponding density. The signal $\Psi$ represents an improved forecast (in terms of the conditional distribution) of the demand $D$, assumed to be nonnegative. In order to provide a uniform treatment in cases of nonnegative demand, we set $f_{D \mid \psi}(x)=0, x \leq 0$. Note also that we use a continuous demand and a continuous signal in this paper for convenience in exposition.

Denote $q_{i}$ as the order quantity in Stage $i, i=1,2$. We can write the buyer's conditional expected cost at Stage 2 as

$$
\begin{aligned}
& G_{2}\left(q_{1}, q_{2} \mid \Psi\right) \\
& = \begin{cases}K+c_{2} q_{2}+E\left[h\left(q_{1}+q_{2}-D\right)^{+}+p\left(D-q_{1}-q_{2}\right)^{+} \mid \Psi\right], & \text { if } q_{2} \neq 0, \\
E\left[h\left(q_{1}-D\right)^{+}+p\left(D-q_{1}\right)^{+} \mid \Psi\right], & \text { if } q_{2}=0,\end{cases}
\end{aligned}
$$

where

$$
c_{2}= \begin{cases}c_{21}, & \text { if } q_{2} \geq 0, \\ c_{22}, & \text { if } q_{2}<0 .\end{cases}
$$

Note that $q_{2}$ is a history-dependent decision variable and $G_{2}\left(q_{1}, q_{2} \mid \Psi\right)$ is a random variable. 
For $\Psi=\psi$, we will write the conditional expected cost, which we note is not a random variable, as

$$
G_{2}\left(q_{1}, q_{2} \mid \psi\right)=G_{2}\left(q_{1}, q_{2} \mid \Psi=\psi\right) .
$$

The buyer's total expected cost is

$$
C\left(q_{1}, q_{2}\right)=c_{1} q_{1}+E\left[G_{2}\left(q_{1}, q_{2} \mid \Psi\right)\right] .
$$

Our purpose is to minimize the total expected cost, i.e., the value function $v_{1}$ is defined as follows:

$$
v_{1}=\min _{q_{1} \geq 0, q_{2} \geq-q_{1}} C\left(q_{1}, q_{2}\right)=\min _{q_{1} \geq 0, q_{2} \geq-q_{1}}\left\{c_{1} q_{1}+E\left[G_{2}\left(q_{1}, q_{2} \mid \Psi\right)\right]\right\} .
$$

The dynamic programming equations for this problem are

$$
\begin{aligned}
v_{2}\left(q_{1}, \psi\right) & =\min _{q_{2} \geq-q_{1}}\left\{G_{2}\left(q_{1}, q_{2} \mid \psi\right)\right\}, \\
v_{1} & =\min _{q_{1} \geq 0}\left\{G_{1}\left(q_{1}\right)\right\},
\end{aligned}
$$

where

$$
G_{1}\left(q_{1}\right)=c_{1} q_{1}+E\left[v_{2}\left(q_{1}, \Psi\right)\right]
$$

\section{Optimal Solution for Stage 2}

In this section we explore structural properties of the second stage cost function in our contract model. First we consider the case when there is no fixed cost of exercising the contract. Thus, with $K=0$, the cost function (3) reduces to

$$
\begin{aligned}
& g_{2}\left(q_{1}, q_{2} \mid \psi\right) \\
= & \begin{cases}g_{2}^{+}\left(q_{1}, q_{2} \mid \psi\right)=c_{21} q_{2}+E\left[h\left(q_{1}+q_{2}-D\right)^{+}+p\left(D-q_{1}-q_{2}\right)^{+} \mid \Psi=\psi\right], & q_{2} \geq 0, \\
g_{2}^{-}\left(q_{1}, q_{2} \mid \psi\right)=c_{22} q_{2}+E\left[h\left(q_{1}+q_{2}-D\right)^{+}+p\left(D-q_{1}-q_{2}\right)^{+} \mid \Psi=\psi\right], & q_{2} \leq 0 .\end{cases}
\end{aligned}
$$

Note that $g_{2}^{+}\left(q_{1}, 0 \mid \psi\right)=g_{2}^{-}\left(q_{1}, 0 \mid \psi\right)=g_{2}\left(q_{1}, 0 \mid \psi\right)$, and therefore $g_{2}\left(q_{1}, q_{2} \mid \psi\right)$ is continuous.

We present the optimal solution in the following lemma.

Lemma 4.1 For $K=0$, the cost function $g_{2}\left(q_{1}, q_{2} \mid \psi\right)$ is convex in $q_{2}$, and differentiable except at $q_{2}=0$ (assuming $c_{21} \neq c_{22}$ ). The optimal adjustment at Stage 2 is

$$
q_{2}^{*}(\psi)= \begin{cases}\Sigma_{1}(\psi)-q_{1}, & \text { if } q_{1}<\Sigma_{1}(\psi), \\ 0, & \text { if } \Sigma_{1}(\psi) \leq q_{1} \leq \Sigma_{2}(\psi), \\ \Sigma_{2}(\psi)-q_{1}, & \text { if } q_{1}>\Sigma_{2}(\psi),\end{cases}
$$


where

$$
0 \leq \Sigma_{1}(\psi)=F_{D \mid \psi}^{-1}\left(\rho_{1}\right)<F_{D \mid \psi}^{-1}\left(\rho_{2}\right)=\Sigma_{2}(\psi),
$$

with

$$
\rho_{i}=\frac{p-c_{2 i}}{p+h}, \quad i=1,2 .
$$

Remark 4.1 Note that $q_{1}$ can be considered to be the inventory level at the beginning of Stage 2 . Then the Stage 2 problem when $q_{2}^{*}(\psi) \geq 0$ is easily seen to be the standard newsvendor problem. The result in Lemma 4.1 could therefore be considered as an extension of the newsvendor problem when returns are allowed.

In Figure 1, we depict the cost $g_{2}\left(q_{1}, q_{2} \mid \psi\right)$ as a function of $q_{2}$ with five different values of $q_{1}$. From (8), we see that each cost curve consists of two pieces: $g_{2}^{-}$for $q_{2} \leq 0$ and $g_{2}^{+}$for $q_{2} \geq 0$. The cost curve $a$ has an interior minimum of $g_{2}^{-}\left(q_{1}, q_{2} \mid \psi\right)$ at $q_{2}<0$. In this case $q_{1}>\Sigma_{2}(\psi)$ and $q_{2}^{*}=\Sigma_{2}(\psi)-q_{1}<0$. When $q_{1}$ decreases to $\Sigma_{2}(\psi)$, we get the cost curve $b$ where the interior minimum of $g_{2}^{-}\left(q_{1}, q_{2} \mid \psi\right)$ is obtained at $q_{2}^{*}=0$. Going to the other side when $q_{1}<\Sigma_{1}(\psi)$, we have cost curve $e$. Here $g_{2}^{+}\left(q_{1}, q_{2} \mid \psi\right)$ takes an interior minimum at $q_{2}^{*}=\Sigma_{1}(\psi)-q_{1}>0$. When $q_{1}$ increases to $\Sigma_{1}(\psi)$, we get the cost curve $d$ where $g_{2}^{+}\left(q_{1}, q_{2} \mid \psi\right)$ has an interior minimum at $q_{2}^{*}=0$. The remaining cost curve $c$ represents the case of a $q_{1}$ such that $\Sigma_{1}(\psi) \leq q_{1} \leq \Sigma_{2}(\psi)$. Here, the minimum is at $q_{2}^{*}=0$. This minimum is a boundary minimum of both $g_{2}^{+}\left(q_{1}, q_{2} \mid \psi\right)$ and $g_{2}^{-}\left(q_{1}, q_{2} \mid \psi\right)$. In fact, the interior minimum of $g_{2}^{-}\left(q_{1}, q_{2} \mid \psi\right)$ would be at some $q_{2}>0$ if it was applicable. Then likewise, the interior minimum of $g_{2}^{+}\left(q_{1}, q_{2} \mid \psi\right)$ would be at $q_{2}<0$ if it was applicable.

We now return to the case when $K>0$. The cost function $G_{2}\left(q_{1}, q_{2} \mid \psi\right)$ is discontinuous at $q_{2}=0$. Consider the difference between the cost of no ordering and that of bringing the inventory level up to $\Sigma_{1}(\psi)$ or down to $\Sigma_{2}(\psi)$ depending on whether $q_{1}<\Sigma_{1}(\psi)$ or $q_{1}>\Sigma_{2}(\psi)$, respectively.

Lemma 4.2 (i) $g_{2}^{+}\left(q_{1}, 0 \mid \psi\right)-K-g_{2}^{+}\left(q_{1}, \Sigma_{1}(\psi)-q_{1} \mid \psi\right)$ is strictly convex and decreasing in $q_{1}$ for all $q_{1}<\Sigma_{1}(\psi)$ and $g_{2}^{-}\left(q_{1}, 0 \mid \psi\right)-K-g_{2}^{-}\left(q_{1}, \Sigma_{2}(\psi)-q_{1} \mid \psi\right)$ is strictly convex and increasing in $q_{1}$ for all $q_{1}>\Sigma_{2}(\psi)$. 


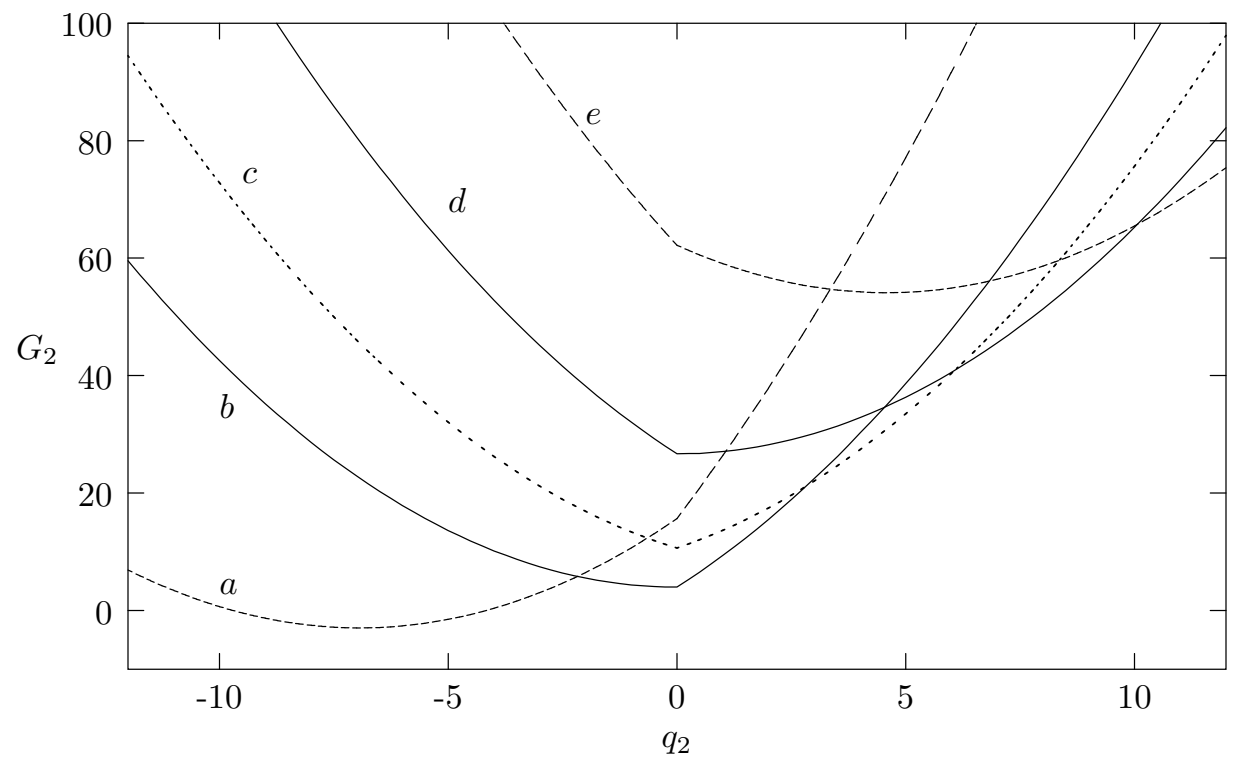

Figure 1: Curves of $G_{2}\left(q_{1}, q_{2}, i\right)$ as a function of $q_{2}$ when $q_{1}$ takes different values. For Curve $a$, $q_{1}=62.0$, for Curve $b, q_{1}=55.1=\Sigma_{2}$, for Curve $c, q_{1}=52.0$, for Curve $d, q_{1}=48.6=\Sigma_{1}$, and for Curve $e, q_{1}=44.0$. Other parameters are $p=10.3, h=0.5, c_{1}=3, c_{21}=6, c_{22}=1$, and $K=5$. The conditional demand distribution given the signal is uniform over the interval $[43,57]$.

(ii) There exist a unique $\sigma_{1}(\psi)<\Sigma_{1}(\psi)$ such that $g_{2}^{+}\left(\sigma_{1}(\psi), 0 \mid \psi\right)=K+g_{2}^{+}\left(\sigma_{1}(\psi), \Sigma_{1}(\psi)-\right.$ $\left.\sigma_{1}(\psi) \mid \psi\right)$ if $\left.K<p+h\right) \int_{0}^{\Sigma_{1}} x f_{D \mid \psi}(x) d x$, and a unique $\sigma_{2}(\psi)>\Sigma_{2}(\psi)$ such that $g_{2}^{-}\left(\sigma_{2}(\psi), 0 \mid \psi\right)=$ $K+g_{2}^{-}\left(\sigma_{2}(\psi), \Sigma_{2}(\psi)-\sigma_{2}(\psi) \mid \psi\right)$.

Based on above preliminaries, we present the main result of this section as follows.

Theorem 4.3 The optimal policy at Stage 2 is

$$
q_{2}^{*}(\psi)= \begin{cases}\Sigma_{1}(\psi)-q_{1}, & \text { if } q_{1}<\sigma_{1}(\psi), \\ 0, & \text { if } \sigma_{1}(\psi) \leq q_{1} \leq \sigma_{2}(\psi), \\ \Sigma_{2}(\psi)-q_{1}, & \text { if } q_{1}>\sigma_{2}(\psi) .\end{cases}
$$

This policy can be thought of as a two-sided $(s, S)$ policy, one for increasing the initial order and the other for decreasing the initial order. We shall term this policy as the $\left[\sigma_{1}(\psi), \Sigma_{1}(\psi) ; \sigma_{2}(\psi), \Sigma_{2}(\psi)\right]$, or more simply, as the $\left(\sigma_{1}, \Sigma_{1} ; \sigma_{2}, \Sigma_{2}\right)(\psi)$ policy. The parameters $\sigma_{1}(\psi)$ and $\Sigma_{1}(\psi)$ are reorder point and order-up-to level, whereas $\sigma_{2}(\psi)$ and $\Sigma_{2}(\psi)$ are reduction point and reduce-down-to level, respectively. In words, the buyer increases the initial order to raise it to $\Sigma_{1}(\psi)$ when the initial order is lower than $\sigma_{1}(\psi)$, the buyer decreases the initial order to reduce it down to $\Sigma_{2}(\psi)$ 
when the initial order is higher than $\sigma_{2}(\psi)$, and the buyer takes no action when the initial order is within the interval $\left[\sigma_{1}(\psi), \sigma_{2}(\psi)\right]$.

The $\left(\sigma_{1}, \Sigma_{1} ; \sigma_{2}, \Sigma_{2}\right)(\psi)$ policy can be considered as a generalized newsvendor problem with a piecewise linear ordering/cancellation cost and a fixed cost. For newsvendor models with piecewise linear cost, with setup cost, or with cancellation, respectively, see Porteus (1990) and references therein.

Remark 4.2 The optimal order-up-to level $\Sigma_{1}(\psi)$ and reduce-down-to level $\Sigma_{2}(\psi)$ do not depend on the fixed contract exercise cost $K$. In contrast, the reorder point $\sigma_{1}(\psi)$ and the reduction point $\sigma_{2}(\psi)$ depend on $K$. Intuitively, the optimal order-up-to and reduce-down-to levels strike a balance between over-ordering and under-ordering, while the reorder and reduction points measure the trade-off between inventory/shortage cost and the fixed contract exercise cost.

Remark 4.3 When $p=c_{21}$ and $h=-c_{22}$, there are multiple optimal policies. If $p=c_{21}$, taking no action when $q_{1}<\Sigma_{1}(\psi)$ is clearly an optimal policy. Likewise, if $h=-c_{22}$, taking no action is optimal when $q_{1}>\Sigma_{2}(\psi)$.

\section{Optimal Solution for a Class of Demand Distributions}

In this section, we obtain the optimal policy under the assumptions that the signal distribution is $\mathrm{PF}_{2}$ (Pólya frequency function of order 2), and the conditional demand distribution given the signal can be parameterized by the signal as its location parameter. For the signal distribution $F_{\Psi}(\cdot)$ and its density $f_{\Psi}(\cdot)$ to be $\mathrm{PF}_{2}$, it must hold that

$$
\frac{f_{\Psi}(x)}{F_{\Psi}(x+y)-F_{\Psi}(x)}
$$

is increasing in $x$ for every fixed $y>0$; see Karlin (1968) and Porteus (2002). These distributions have been found to be quite useful, and thus, popular in the inventory literature. Moreover, the class of $\mathrm{PF}_{2}$ densities include a large number of standard unimodal densities arising in inventory theory, such as the exponential, the normal, the truncated normal, the uniform, the gamma, the Erlang, and their convolutions. Note that if we were to use discrete demands, then distributions in the Poisson and the Pascal family qualify as discrete Pólya frequency functions (Schoenberg, 1953). 
For a given family of continuous distributions, there are usually several alternative ways to define, or parameterize the density function. Some distributions can be parameterized by a location parameter, which specifies an abscissa location point of a distribution's range of values. Usually the location point is the midpoint such as the mean for a normal distribution or the lower endpoint in the case of a uniform distribution. See Law and Kelton (2000) for examples of other distributions that can be defined by their location parameters.

Since we assume the signal $\psi$ to be the location parameter of $F_{D \mid \psi}(\cdot)$ and $f_{D \mid \psi}(\cdot)$, it is clear that for $\psi_{2} \geq \psi_{1}$, we have

$$
\begin{aligned}
& f_{D \mid \psi_{2}}(\eta)=f_{D \mid \psi_{1}}\left(\eta-\psi_{2}+\psi_{1}\right) \\
& F_{D \mid \psi_{2}}(\eta)=F_{D \mid \psi_{1}}\left(\eta-\psi_{2}+\psi_{1}\right)
\end{aligned}
$$

In writing (14) and (15), we understand that $F_{D \mid \psi}(x)=f_{D \mid \psi}(x)=0, x \leq 0$, in view of the fact that $D \geq 0$. We can now prove the following result.

Theorem 5.1 For any integrable function $r(x, \eta)$,

$$
E\left[r(x, D) \mid \psi_{2}\right]=E\left[r\left(x, D+\psi_{2}-\psi_{1}\right) \mid \psi_{1}\right]
$$

Furthermore, let $r(x, D)$ be of the form $r(x-D)$ with a slight abuse of notation, and define $g(x \mid \psi)=E[r(x-D) \mid \psi]$. Then,

$$
g\left(x \mid \psi_{2}\right)=E\left[r(x-D) \mid \psi_{2}\right]=E\left[r\left(x-D-\psi_{2}+\psi_{1}\right) \mid \psi_{1}\right]=g\left(x-\psi_{2}+\psi_{1} \mid \psi_{1}\right) .
$$

Relation (17) says that the value of a function $g\left(\cdot \mid \psi_{2}\right)$ at a point $x$ given $\psi_{2}$ can be obtained by evaluating the function $g\left(\cdot \mid \psi_{1}\right)$ at the point $x-\psi_{2}+\psi_{1}$. Geometrically speaking, $g\left(\cdot \mid \psi_{2}\right)$ is nothing but the function $g\left(\cdot \mid \psi_{1}\right)$ shifted to the right by an amount $\psi_{2}-\psi_{1}$. This immediately gives us the following corollary.

Corollary 5.2 The optimal order-up-to level $\Sigma_{1}(\cdot)$ and reduce-down-to level $\Sigma_{2}(\cdot)$ satisfy $\Sigma_{i}\left(\psi_{2}\right)=$ $\Sigma_{i}\left(\psi_{1}\right)+\psi_{2}-\psi_{1}$ for any $\psi_{2} \geq \psi_{1}, i=1,2$. Similarly, the reorder and reduction points satisfy

$$
\sigma_{i}\left(\psi_{2}\right)=\sigma_{i}\left(\psi_{1}\right)+\psi_{2}-\psi_{1}, \quad \text { for } \psi_{2} \geq \psi_{1}, \quad i=1,2 \text {. }
$$


Corollary 5.2 implies that both the levels and points can be expressed as the summation of the location parameter $\psi$ and a constant term which is independent of $\psi$. That is,

$$
\Sigma_{i}(\psi)=\psi+u_{i}, \quad \sigma_{i}(\psi)=\psi+w_{i}, \quad i=1,2,
$$

where $u_{i}=\Sigma_{i}(0)$ and $w_{i}=\sigma_{i}(0)$.

Based on the optimal policy (12) at Stage 2 and using (19), we write the optimal cost function at Stage 2 as follows:

$$
v_{2}\left(q_{1}, \psi\right)= \begin{cases}K+g_{2}^{+}\left(q_{1}, \Sigma_{1}(\psi)-q_{1} \mid \psi\right), & \text { if } \psi>q_{1}-u_{1} \\ g_{2}\left(q_{1}, 0 \mid \psi\right), & \text { if } q_{1}-u_{2} \leq \psi \leq q_{1}-u_{1} \\ K+g_{2}^{-}\left(q_{1}, \Sigma_{2}(\psi)-q_{1} \mid \psi\right), & \text { if } \psi<q_{1}-u_{2}\end{cases}
$$

From the definition of $\sigma_{i}(\psi)$ in Lemma 4.2 and the fact noted in connection with (8), we can see that $v_{2}\left(q_{1}, \psi\right)$ is a continuous function of $\psi$. Using $(20)$ in (7), we obtain

$$
\begin{aligned}
G_{1}\left(q_{1}\right)= & c_{1} q_{1}+\int_{0}^{q_{1}-u_{2}}\left[K+g_{2}^{-}\left(q_{1}, \Sigma_{2}(\psi)-q_{1} \mid \psi\right)\right] f_{\Psi}(\psi) d \psi+\int_{q_{1}-u_{2}}^{q_{1}-u_{1}} g_{2}\left(q_{1}, 0 \mid \psi\right) f_{\Psi}(\psi) d \psi \\
& +\int_{q_{1}-u_{1}}^{\infty}\left[K+g_{2}^{+}\left(q_{1}, \Sigma_{1}(\psi)-q_{1} \mid \psi\right)\right] f_{\Psi}(\psi) d \psi
\end{aligned}
$$

Its derivative with respect to $q_{1}$ as shown in the appendix is

$$
\frac{\partial G_{1}\left(q_{1}\right)}{\partial q_{1}}=c_{1}-\int_{0}^{\infty} c_{2} f_{\Psi}(\psi) d \psi+\int_{q_{1}-u_{2}}^{q_{1}-u_{1}}\left[(p+h) F_{D \mid \psi}\left(q_{1}\right)-\left(p-c_{21}\right)\right] f_{\Psi}(\psi) d \psi
$$

We can now state the main result of this section.

Theorem 5.3 There exists an initial order quantity $q_{1}^{*}$ that minimizes $G_{1}\left(q_{1}\right)$. The total expected cost $G_{1}\left(q_{1}\right)$ is a unimodal function of $q_{1}$, and the optimal initial order quantity $q_{1}^{*}$ satisfies

$$
c_{1}-\int_{0}^{\infty} c_{2} f_{\Psi}(\psi) d \psi+\int_{q_{1}^{*}-u_{2}}^{q_{1}^{*}-u_{1}}\left[(p+h) F_{D \mid \psi}\left(q_{1}^{*}\right)-\left(p-c_{21}\right)\right] f_{\Psi}(\psi) d \psi=0 .
$$

Furthermore, if the signal distribution has a location parameter $\gamma$, then the optimal initial order quantity satisfies

$$
q_{1}^{*}\left(\gamma_{2}\right)=q_{1}^{*}\left(\gamma_{1}\right)+\gamma_{2}-\gamma_{1}, \quad \forall \gamma_{2} \geq \gamma_{1}
$$




\section{Further Analysis with a Uniformly Distributed Demand}

To facilitate further analysis, we assume a uniformly distributed signal $\Psi$ over the interval $[\gamma-$ $\left.\frac{a}{2}, \gamma+\frac{a}{2}\right]$. We further assume that the demand $D$ follows the uniform distribution over the interval $\left[\psi-\frac{\varepsilon a}{2}, \psi+\frac{\varepsilon a}{2}\right]$, given $\Psi=\psi$. Thus,

$$
\begin{array}{ll}
f_{\Psi}(\psi)=\frac{1}{a}, & \psi \in\left[\gamma-\frac{a}{2}, \gamma+\frac{a}{2}\right], \\
f_{D \mid \psi}(\eta)=\frac{1}{\varepsilon a}, & \eta \in\left[\psi-\frac{\varepsilon a}{2}, \psi+\frac{\varepsilon a}{2}\right],
\end{array}
$$

where $0 \leq \varepsilon \leq 1$ represents the reduction in the forecast errors at the updating stage. The value of $\varepsilon$ can be obtained from either the buyer's experience or regression methods (Yan, Liu and Hsu, 2003).

In this case, the results obtained in previous sections yield explicit formulas for the cost functions $G_{1}\left(q_{1}\right), G_{2}\left(q_{1}, q_{2} / \psi\right), v_{1}$ and $v_{2}\left(q_{1}, \psi\right)$ and the order quantities $q_{2}^{*}(\psi)$ and $q_{1}^{*}$, after some tedious, albeit straightforward, calculations. We do not write these expressions here because of a large number of cases involved. See Huang (2002) for details.

Yan, Liu and Hsu (2003) have studied a two-stage purchase problem in the same local security equipment company as mentioned in Section 1. The company purchases low-priced masking chips and high priced generic chips in Stage 1 and Stage 2, respectively. Using the same distribution assumptions as in (25), they obtained the expected cost functions

$$
\begin{aligned}
\widetilde{G}_{2}\left(q_{1}, q_{2} \mid \psi\right) & =\widetilde{c}_{2} q_{2}+h \int_{\psi-\varepsilon a / 2}^{q_{1}+q_{2}}\left(q_{1}+q_{2}-\eta\right) f_{D \mid \psi}(\eta) d \eta+p \int_{q_{1}+q_{2}}^{\psi+\varepsilon a / 2}\left(\eta-q_{1}-q_{2}\right) f_{D \mid \psi}(\eta) d \eta \\
\widetilde{G}_{1}\left(q_{1}\right) & =c_{1} q_{1}+E\left[\widetilde{G}_{2}\left(q_{1}, q_{2} \mid \Psi\right)\right]
\end{aligned}
$$

for Stage 2 and Stage 1, respectively, where $\widetilde{c}_{2}$ is the purchase cost of a single generic component. With $G_{1}\left(q_{1}\right)$ and the expression (26) in hand, it is straightforward to derive the following results.

Theorem 6.1 With (25), the contract cost function $G_{1}\left(q_{1}\right)$ in $(21)$ and the substitution cost function $\widetilde{G}_{1}\left(q_{1}\right)$ exhibit the following properties:

(i) $\min _{q_{1} \geq 0}\left\{G_{1}\left(q_{1}\right)\right\}$ is a monotone nondecreasing function with respect to the contract exercise cost $K$;

(ii) $\min _{q_{1} \geq 0}\left\{G_{1}\left(q_{1}\right) \mid K=0\right\} \leq \min _{q_{1} \geq 0}\left\{\widetilde{G}_{1}\left(q_{1}\right)\right\} \leq \min _{q_{1} \geq 0}\left\{G_{1}\left(q_{1}\right) \mid K<(p+h) \int_{0}^{\Sigma_{1}} x f_{D \mid \psi}(x) d x\right\}$; and 
(iii) there exists a unique $K_{1}$ such that $\min _{q_{1} \geq 0}\left\{G_{1}\left(q_{1}\right) \mid K=K_{1}\right\}=\min _{q_{1} \geq 0}\left\{\widetilde{G}_{1}\left(q_{1}\right)\right\}$.

Theorem 6.1(iii) reveals a rule for hedging strategy selection. When $K \geq K_{1}$, it is unwise for the buyer to sign the flexible purchase contract. By noting that a purchase contract is a real option, $K$ can be considered as the option price in the case when $c_{21}=c_{22}=c_{1}$. It is of interest for the buyer to know the value of the purchase contract. In particular, the buyer needs to know what is the best strategy against demand uncertainty.

Once we have an expression for $v_{1}$, it is possible to carry out a sensitivity analysis with respect to forecast and contract parameters. For example, with the assumption (25), $v_{1}$ turns out to be a function of the parameters $\varepsilon$ and $a$. By differentiating $v_{1}$ with respect to these parameters, we obtain the corresponding marginal benefits, $-\frac{\partial v_{1}}{\partial \varepsilon}$, and $-\frac{\partial v_{1}}{\partial a}$, which provide an indication of the relative importance of the Stage 1 and the Stage 2 forecasts in the model, respectively. Given these and the costs of efforts in reducing $a$ and $\varepsilon$, the buyer's company can figure out where it should put it's next dollar in improving the demand forecast.

As for the contract exercise cost $K$, it is possible to identify a critical value $K_{0}$, such that if $K$ is more than (resp. less than) $K_{0}$, then the buyer should invest in improving Stage 1 (resp. Stage 2) forecast. To further illustrate the sensitivity of the optimal decision with respect to the model parameters $a, \varepsilon$ and $K$, Figures 2, 3 and 4 plotted for $p=10, h=0.3, c_{1}=1$, and $c_{2}=3$, depict the cost functions with respect to $a, \varepsilon$ and $K$, respectively. Figure 2 plotted for $\varepsilon=0.75$ and $K=20$ illustrates that the optimal cost is an increasing function of the demand spread. Figure 3 plotted for $a=20$ and $K=20$ illustrates that the optimal cost is a decreasing function of the reduction in the forecast error. Figure 4 plotted for $a=20$ and $\varepsilon=0.75$ illustrates that the optimal cost is an increasing function of the setup cost.

\section{Conclusions}

In this paper we study a purchase contract with a demand forecast update. We formulate the problem as a two-stage dynamic programming problem. We obtain an optimal solution for the contract management for the class of $\mathrm{PF}_{2}$ demand distributions. In particular, we obtain an explicit optimal solution for a uniformly distributed updated demand. The explicit nature of the 


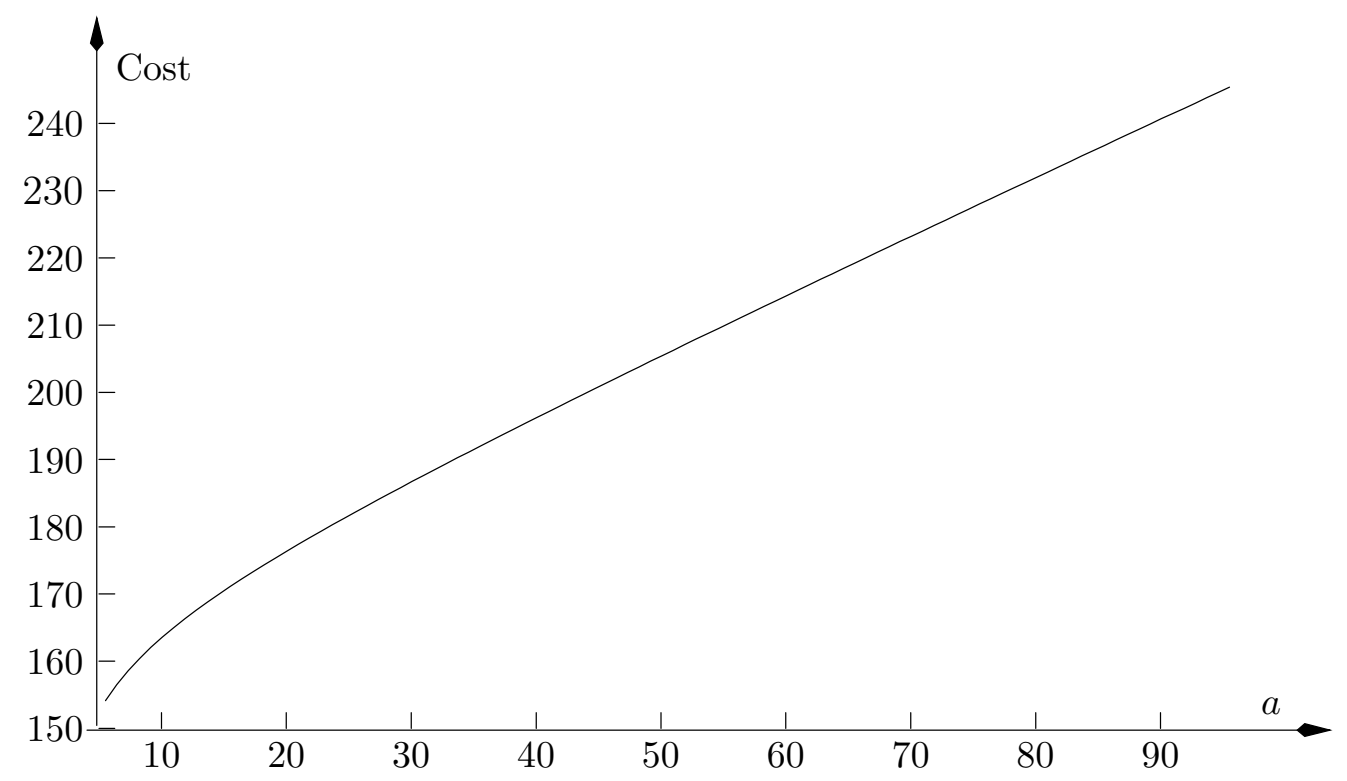

Figure 2: The Optimal cost with respect to the spread of the demand distribution

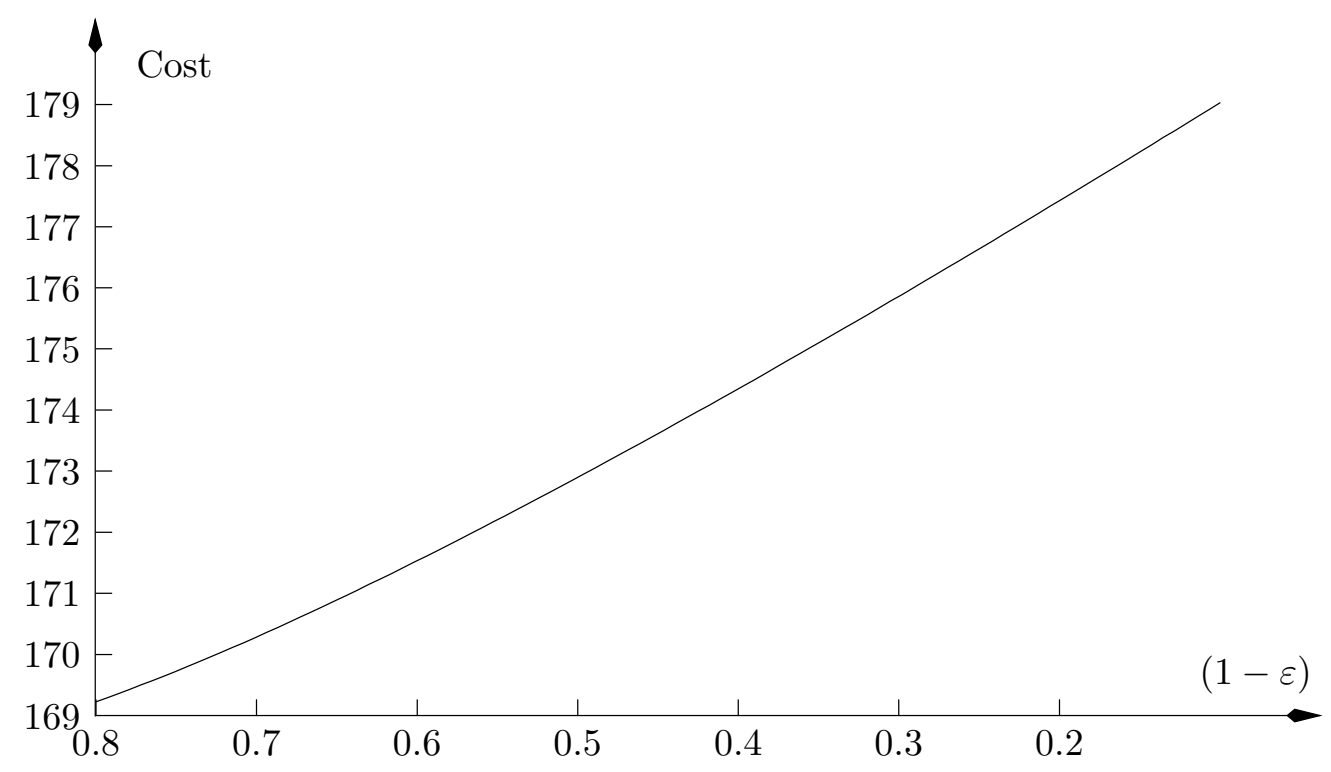

Figure 3: The optimal cost with respect to the reduction of forecast errors 


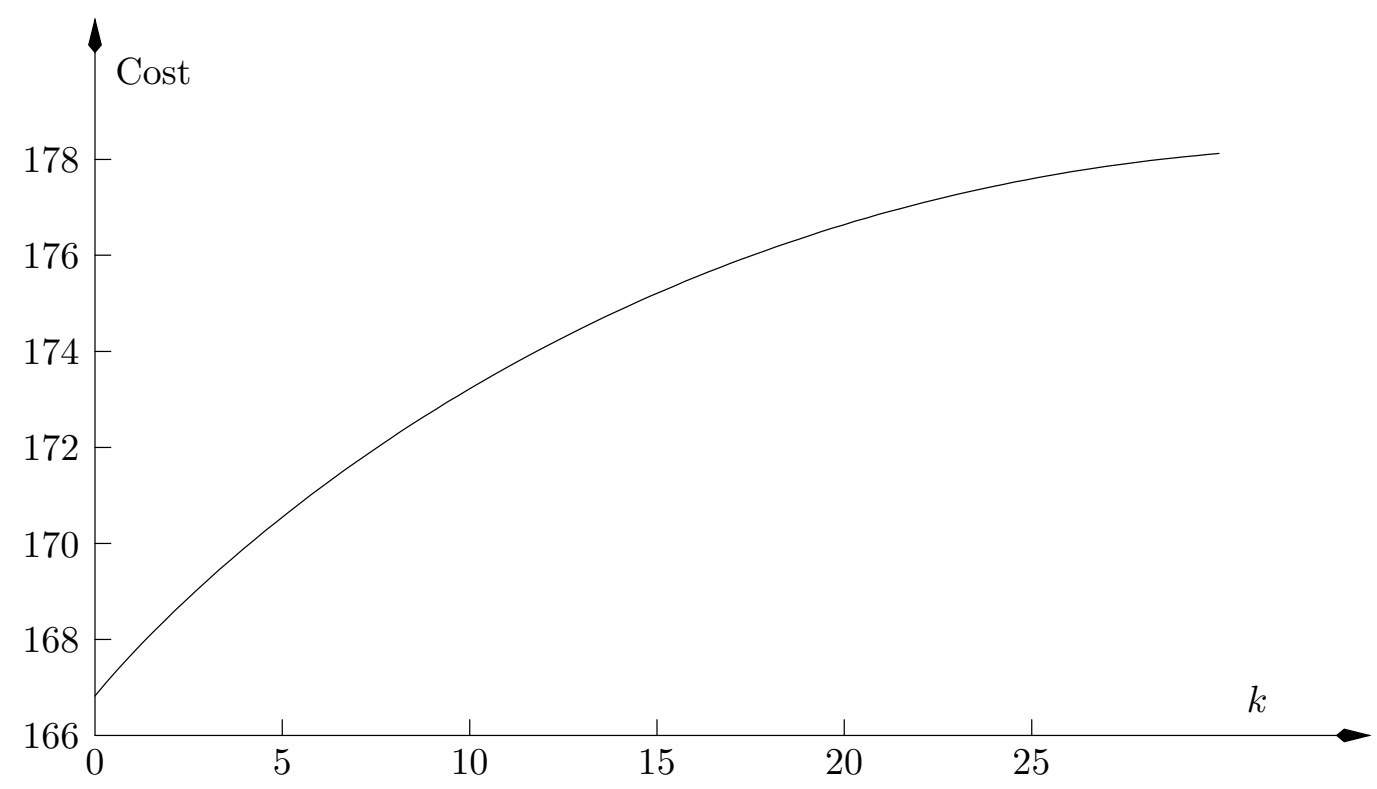

Figure 4: the optimal cost with respect to the setup cost

optimal solution enables us to find a critical value of the contract exercise cost, which determines the direction of further improvement in the demand forecast. In comparison with other risk hedging approaches such as substitution, we obtain a critical value of the contract exercise cost, below (resp. above) which the buyer would (resp. would not) sign the contract.

\section{Acknowledgements}

This research was supported in part by Research Grant RGC CUHK4239/03E and NSFC/RGC CUHK10. The authors would like to thank the AE and two referees for detailed comments and suggestions.

\section{Appendix}

\section{Proof of Lemma 4.1.}

The convexity of $g_{2}\left(q_{1}, q_{2} \mid \psi\right)$ in $q_{2}$ and its non-differentiability at $q_{2}=0$ are obvious. For $q_{2}>0$, setting

$$
\frac{\partial g_{2}^{+}}{\partial q_{2}}=\left(c_{21}-p\right)+(p+h) F_{D \mid \psi}\left(q_{1}+q_{2}\right)=0 .
$$

provides the minimum $\Sigma_{1}(\psi)$ as specified in (10) and (11), as $c_{21} \geq c_{1} \geq c_{22}$. Likewise, for $q_{2}<0$, using $g_{2}^{-}$instead of $g_{2}^{+}$in (27) gives us $\Sigma_{2}(\psi) \geq \Sigma_{1}(\psi)$ as specified in (10) and (11). Also, it is obvious $\Sigma_{1}(\psi) \geq 0$ as $q_{2} \geq-q_{1}$. Clearly then, the policies delivered for $q_{1}<\Sigma_{1}(\psi)$ and 
$q_{1}>\Sigma_{2}(\psi)$ in (9) are optimal. Furthermore, when $\Sigma_{1}(\psi) \leq q_{1} \leq \Sigma_{2}(\psi)$, the optimality condition in this case, because of the non-differentiability at $q_{2}=0$, is

$$
0 \in\left[\left(c_{22}-p\right)+(p+h) F_{D \mid \psi}\left(q_{1}+q_{2}\right),\left(c_{21}-p\right)+(p+h) F_{D \mid \psi}\left(q_{1}+q_{2}\right)\right],
$$

which clearly holds when $q_{1} \in\left[\Sigma_{1}(\psi), \Sigma_{2}(\psi)\right]$.

\section{Proof of Lemma 4.2.}

Define

$$
\Delta\left(q_{1} \mid \psi\right)= \begin{cases}g_{2}^{+}\left(q_{1}, 0 \mid \psi\right)-K-g_{2}^{+}\left(q_{1}, \Sigma_{1}(\psi)-q_{1} \mid \psi\right), & q_{1}<\Sigma_{1}(\psi), \\ g_{2}^{-}\left(q_{1}, 0 \mid \psi\right)-K-g_{2}^{-}\left(q_{1}, \Sigma_{2}(\psi)-q_{1} \mid \psi\right), & q_{1}>\Sigma_{2}(\psi),\end{cases}
$$

to be the difference between the cost of no ordering and that of bringing the inventory level up to $\Sigma_{1}(\psi)$ or down to $\Sigma_{2}(\psi)$ depending on whether $q_{1}<\Sigma_{1}(\psi)$ or $q_{1}>\Sigma_{2}(\psi)$, respectively.

(i) Since $g_{2}^{+}\left(q_{1}, 0 \mid \psi\right)$ is strictly convex in $q_{1}$ and $g_{2}^{+}\left(q_{1}, \Sigma_{1}(\psi)-q_{1} \mid \psi\right)$ is linear in $q_{1}, \Delta\left(q_{1} \mid \psi\right)$ is strictly convex in $q_{1}$ for $q_{1}<\Sigma_{1}(\psi)$. Similarly, $\Delta\left(q_{1} \mid \psi\right)$ is strictly convex in $q_{1}$ for $q_{1}>\Sigma_{2}(\psi)$. Differentiating $\Delta\left(q_{1} \mid \psi\right)$ with respect to $q_{1}$ gives

$$
\frac{d \Delta\left(q_{1} \mid \psi\right)}{d q_{1}}=(p+h) F_{D \mid \psi}\left(q_{1}\right)-\left(p-c_{2}\right)
$$

For all $q_{1}<\Sigma_{1}(\psi), F_{D \mid \psi}\left(q_{1}\right) \leq F_{D \mid \psi}\left[\Sigma_{1}(\psi)\right]=\left(p-c_{21}\right) /(p+h)$, and for all $q_{1}>\Sigma_{2}(\psi), F_{D \mid \psi}\left(q_{1}\right) \geq$ $F_{D \mid \psi}\left[\Sigma_{2}(\psi)\right]=\left(p-c_{22}\right) /(p+h)$. Thus, when $q_{1}<\Sigma_{1}(\psi), \frac{d \Delta\left(q_{1} \mid \psi\right)}{d q_{1}} \leq \frac{d \Delta\left(\Sigma_{1} \mid \psi\right)}{d q_{1}}=0$, and when $q_{1}>\Sigma_{2}(\psi)$, the reverse is true.

(ii) From (29),

$$
\lim _{q_{1} \uparrow \Sigma_{1}(\psi)} \Delta\left(q_{1} \mid \psi\right)=-K<0 .
$$

Also, note that

$$
\begin{aligned}
& \lim _{q_{1} \downarrow 0} \Delta\left(q_{1} \mid \psi\right) \\
= & E\left[h(-D)^{+}+p(D)^{+} \mid \psi\right]-K-c_{21} \Sigma_{1}-E\left[h\left(\Sigma_{1}-D\right)^{+}+p\left(D-\Sigma_{1}\right)^{+} \mid \psi\right] \\
= & (p+h) \int_{0}^{\Sigma_{1}} x f_{D \mid \psi}(x) d x-K
\end{aligned}
$$

is positive when $K<p+h) \int_{0}^{\Sigma_{1}} x f_{D \mid \psi}(x) d x$. In view of the fact that $\Delta\left(q_{1} \mid \psi\right)$ is decreasing in $q_{1}$ for $q_{1}<\Sigma_{1}(\psi)$, there exists a $\sigma_{1}(\psi)$ as stipulated in the statement (ii) of the lemma.

The proof of (ii) for the existence of the required $\sigma_{2}(\psi)$ is similar. Here our assumption of $h>-c_{22}$ implies that $\Delta\left(q_{1} \mid \psi\right) \rightarrow \infty$ as $q_{1} \rightarrow \infty$. 


\section{Proof of Theorem 4.3.}

The proof requires number of cases to be dealt with. Recall that $g_{2}\left(q_{1}, 0 \mid \psi\right)=g_{2}^{+}\left(q_{1}, 0 \mid \psi\right)=$ $g_{2}^{-}\left(q_{1}, 0 \mid \psi\right)$.

Case (1) $q_{1}<\Sigma_{1}(\psi)$ : By Lemma 4.1,

$$
\begin{aligned}
& \min \left\{K+\min _{q_{2}<0}\left\{g_{2}^{-}\left(q_{1}, q_{2} \mid \psi\right)\right\}, \quad g_{2}\left(q_{1}, 0 \mid \psi\right), \quad K+\min _{q_{2}>0}\left\{g_{2}^{+}\left(q_{1}, q_{2} \mid \psi\right)\right\}\right\} \\
= & \min \left\{K+g_{2}^{-}\left(q_{1}, 0 \mid \psi\right), \quad g_{2}\left(q_{1}, 0 \mid \psi\right), \quad K+g_{2}^{+}\left(q_{1}, \Sigma_{1}(\psi)-q_{1} \mid \psi\right)\right\} \\
= & \min \left\{g_{2}^{+}\left(q_{1}, 0 \mid \psi\right), \quad K+g_{2}^{+}\left(q_{1}, \Sigma_{1}(\psi)-q_{1} \mid \psi\right)\right\} .
\end{aligned}
$$

By (ii) of Lemma 4.2, when $q_{1}<\sigma_{1}(\psi), g_{2}^{+}\left(q_{1}, 0 \mid \psi\right)>K+g_{2}^{+}\left(q_{1}, \Sigma_{1}(\psi)-q_{1} \mid \psi\right)$. Thus $q_{2}^{*}(\psi)=$ $\Sigma_{1}(\psi)-q_{1}$. When $q_{1} \geq \sigma_{1}(\psi)$, we have $g_{2}^{+}\left(q_{1}, 0 \mid \psi\right) \leq K+g_{2}^{+}\left(q_{1}, \Sigma_{1}(\psi)-q_{1} \mid \psi\right)$, and therefore $q_{2}^{*}(\psi)=0$.

Case (2) $q_{1}>\Sigma_{2}(\psi)$ : Analogous reasoning as in Case (1).

Case $(3) \Sigma_{1}(\psi) \leq q_{1} \leq \Sigma_{2}(\psi)$ : By Lemma 4.1,

$$
\begin{aligned}
& \min \left\{K+\min _{q_{2}<0}\left\{g_{2}^{-}\left(q_{1}, q_{2} \mid \psi\right)\right\}, \quad g_{2}\left(q_{1}, 0 \mid \psi\right), \quad K+\min _{q_{2}>0}\left\{g_{2}^{+}\left(q_{1}, q_{2} \mid \psi\right)\right\}\right\} \\
= & \min \left\{K+g_{2}^{-}\left(q_{1}, 0 \mid \psi\right), \quad g_{2}\left(q_{1}, 0 \mid \psi\right), \quad K+g_{2}^{+}\left(q_{1}, 0 \mid \psi\right)\right\} \\
= & g_{2}\left(q_{1}, 0 \mid \psi\right) .
\end{aligned}
$$

Thus $q_{2}^{*}(\psi)=0$.

\section{Proof of Theorem 5.1.}

Using (14), we have

$$
\begin{aligned}
E\left[r(x, D) \mid \psi_{2}\right] & =\int_{-\infty}^{+\infty} r(x, \eta) f_{D \mid \psi_{2}}(\eta) d \eta=\int_{-\infty}^{+\infty} r(x, \eta) f_{D \mid \psi_{1}}\left(\eta-\psi_{2}+\psi_{1}\right) d \eta \\
& =\int_{-\infty}^{+\infty} r\left(x, \eta+\psi_{2}-\psi_{1}\right) f_{D \mid \psi_{1}}(\eta) d \eta=E\left[r\left(x, D+\psi_{2}-\psi_{1}\right) \mid \psi_{1}\right] .
\end{aligned}
$$

The proof of the second part follows trivially from the first.

Derivation of (22).

$$
\begin{aligned}
\frac{\partial G_{1}\left(q_{1}\right)}{\partial q_{1}}= & c_{1}+\left.\left[K+g_{2}^{-}\left(q_{1}, \Sigma_{2}(\psi)-q_{1} \mid \psi\right)\right] f_{\Psi}(\psi)\right|_{\psi=q_{1}-u_{2}}-\int_{0}^{q_{1}-u_{2}} c_{22} f_{\Psi}(\psi) d \psi \\
& +\left.g_{2}\left(q_{1}, 0 \mid \psi\right) f_{\Psi}(\psi)\right|_{\psi=q_{1}-u_{2}} ^{q_{1}-u_{1}}+\int_{q_{1}-u_{2}}^{q_{1}-u_{1}}\left[(p+h) F_{D \mid \psi}\left(q_{1}\right)-p\right] f_{\Psi}(\psi) d \psi
\end{aligned}
$$




$$
\begin{aligned}
& -\left.\left[K+g_{2}^{+}\left(q_{1}, \Sigma_{1}(\psi)-q_{1} \mid \psi\right)\right] f_{\Psi}(\psi)\right|_{\psi=q_{1}-u_{1}}-\int_{q_{1}-u_{1}}^{\infty} c_{21} f_{\Psi}(\psi) d \psi \\
= & c_{1}-\int_{0}^{\infty} c_{2} f_{\Psi}(\psi) d \psi+\int_{q_{1}-u_{2}}^{q_{1}-u_{1}}\left[(p+h) F_{D \mid \psi}\left(q_{1}\right)-\left(p-c_{21}\right)\right] f_{\Psi}(\psi) d \psi \\
& +\left.\Delta\left(q_{1} \mid \psi\right) f_{\Psi}(\psi)\right|_{\psi=q_{1}-u_{1}}-\left.\Delta\left(q_{1} \mid \psi\right) f_{\Psi}(\psi)\right|_{\psi=q_{1}-u_{2}} \\
= & c_{1}-\int_{0}^{\infty} c_{2} f_{\Psi}(\psi) d \psi+\int_{q_{1}-u_{2}}^{q_{1}-u_{1}}\left[(p+h) F_{D \mid \psi}\left(q_{1}\right)-\left(p-c_{21}\right)\right] f_{\Psi}(\psi) d \psi,
\end{aligned}
$$

where the second equality uses the definitions of $c_{2}$ and $\Delta\left(q_{1} \mid \psi\right)$, and the last equality is obtained by noting that $\left.\Delta\left(q_{1} \mid \psi\right)\right|_{\psi=q_{1}-u_{i}}=\Delta\left(\sigma_{i}(\psi) \mid \psi\right)=0$ for $i=1,2$.

\section{Proof of Theorem 5.3.}

(1) The existence of $q_{1}^{*}$ follows from the continuity of $v_{2}\left(q_{1}, \psi\right)$ and the facts that $\lim _{q_{1} \rightarrow 0} \partial G_{1} / \partial q_{1}=$ $c_{1}-\int_{0}^{\infty} c_{21} f_{\Psi}(\psi) d \psi=c_{1}-c_{21}<0$ and $\lim _{q_{1} \rightarrow \infty} G_{1}\left(q_{1}\right)=\infty$.

(2) By (20), it is easy to see that $v_{2}\left(q_{1}, \psi\right)$ is unimodal in $q_{1}$. Recall that $G_{1}\left(q_{1}\right)=c_{1} q_{1}+$ $E\left[v_{2}\left(q_{1}, \Psi\right)\right]$, and it is known from Schoenberg (1951) or Karlin (1968) that the expectation of a unimodal function under a $\mathrm{PF}_{2}$ density is still a unimodal function.

(3) The proof of (24) is along the lines of Theorem 5.1 and Corollary 5.2.

\section{Proof of Theorem 6.1}

(i) We can show that the optimal reordering point $\sigma_{1}=\Sigma_{1}-\mu(K)$, the reducing point

$\sigma_{2}=\Sigma_{2}+\mu(K)$ and $\mu(K)=\sqrt{\frac{2 \varepsilon a K}{\alpha_{1}}}$. The derivation is simple, but tedious so it is omitted. Therefore, $\sigma_{1}$ decreases, $\sigma_{2}$ increases when $K$ increases. The feasible set of $q_{2}$ shrinks when $K$ increases. Therefore, $\min _{q_{1}}\left\{G_{1}\left(q_{1}\right)\right\}$ is a monotone non-decreasing function of $K$.

(ii) The validity of the left hand side inequality lies in that the feasible set of $q_{2}$ becomes larger when negative $q_{2}$ is allowed; In addition, a sufficient large contract price $k$ forces the initial order quantity to remain unchanged. This fact results in the validity of the right hand side inequality.

(iii) Properties (i) and (ii) ensure the existence of $K_{1}$.

\section{References}

[1] Anupindi, R. and Y. Bassok, "Approximations for multiproduct contracts with stochastic demands and business volume discounts: single supplier case," IIE Transactions, Vol. 30, No. 8, 723-734, 1998. 
[2] Barnes-Schuster, D., Y. Bassok, and R. Anupindi, "Coordination and flexibility in supply contracts with options," Manufacturing 8 Service Operations Management, Vol. 4, No. 3, 171-207, 2002.

[3] Bassok Y. and R. Anupindi, "Analysis of supply contracts with total minimum commitment," IIE Transactions, Vol. 29, No. 5, 373-382, 1997.

[4] Bazaraa, M. S., H. D. Sherali, and C. M. Shetty, Nonlinear Programming: Theory and Algorithms, Wiley, New York, NY, 1993.

[5] Cachon, G. P., "Supply Chain Coordination with Contracts," A. G. de Kok, S. C. Graves, eds, Handbooks in Operations Research and Mangement Science, Volume 11: Supply Chain Management: Design, Coordination and Operation, Elsevier, Amsterdam, 229-339, 2003.

[6] Donohue, K. L., "Efficient supply contracts for fashion goods with forecast updating and two production modes," Management Science, Vol. 46, No. 11, 1397 - 1411, 2000.

[7] Eppen, G. D. and A. V. Iyer, "Backup agreements in fashion buying - the value of upstream flexibility," Management Science, Vol. 43, No. 11, 1469-1484, 1997.

[8] Fisher, M. and A. Raman, "Reducing the cost of demand uncertainty through accurate response to early sales," Operations Research, Vol. 44, No. 1, 87-99, 1996.

[9] Gurnani, H. and C. S. Tang, "Note: optimal ordering decisions with uncertain cost and demand forecast updating," Management Science, Vol. 45, No. 10, 1456-1462, 1999.

[10] Huang, H. Y., "Demand information updating and sharing in purchase contract management," Ph.D. Dissertation, The Chinese University of Hong Kong, 2002.

[11] Iyer, A. V. and M. E. Bergen, "Quick response in manufacturer- retailer channels," Management Science, Vol. 43, No. 4, 559-570, 1997.

[12] Karlin, S., Total Positivity, Vol. 1, Stanford University Press, Stanford, CA, 1968.

[13] Law, A. M. and W. D. Kelton, Simulation Modeling and Analysis, 3rd Edition, McGraw Hill, New York, NY, 2000. 
[14] Lovejoy, W., "Myopic policies for some inventory models with uncertain demand distributions," Management Science, Vol. 36, No. 6, 724-738, 1990.

[15] Murray, G. R. and E. A. Silver, "A Bayesian analysis of the style goods inventory problem," Management Science, Vol. 12, No. 11, 785-797, 1966.

[16] Porteus, E. L., Foundations of Stochastic Inventory Theory, Stanford University Press, Stanford, CA, 2002.

[17] —, "Stochastic Inventory Theory," D. P. Heyman and M. J. Sobel, eds, Handbook in Operations Research and Management Science, Volume 2: Stochastic Models, North-Holland, Amsterdam, The Netherlands, 605-652, 1990.

[18] Schoenberg, I., " On Pólya frequency functions I. The totally positive Laplace transforms," Journal d'Analyse Mathématique, Vol. 1, 331-374, 1951.

[19] Schoenberg, I., " On smoothing operations and their generating functions," Bulletin of the American Mathematical Society, Vol. 59, 199-230, 1953.

[20] Sethi, S. and G. Sorger, "A theory of rolling horizon decision making," Annals of Operations Research, Vol. 29, 387-416, 1991.

[21] Sethi, S., H. Yan, and H. Zhang, "Peeling layers of an Onion: Inventory model with multiple delivery modes and forecast updates," Journal of Optimization Theory and Applications, Vol. 108, No. 2, 253-281, 2001.

[22] Sethi, S., H. Yan, and H. Zhang, "Inventory models with fixed costs, forecast updates, and two delivery modes," Operations Research, Vol. 51, No. 2, 321-328, 2003.

[23] Yan H., K. Liu, and A. Hsu, "Optimal ordering in dual-supplier system with demand forecast updates," Production and Operations Management, Vol. 12, 30-45, 2003. 